\title{
Specific lon Binding at Phospholipid Membrane Surfaces
}

\author{
Jing Yang, ${ }^{\dagger}$ Carles Calero, ${ }^{\ddagger}$ Massimiliano Bonomi, ${ }^{,}, \uparrow$ and Jordi Martín ${ }^{*} \dagger$ \\ Department of Physics and Nuclear Engineering, Technical University of \\ Catalonia-Barcelona Tech, B4-B5 Northern Campus, Jordi Girona 1-3, 08034 Barcelona, \\ Catalonia, Spain, Center for Polymer Studies and Department of Physics, Boston \\ University, 590 Commonwealth Avenue, Boston, MA 02215, USA, and Department of \\ Chemistry, University of Cambridge, Lensfield Road, Cambridge CB2 1EW, United \\ Kingdom
}

E-mail: mb2006@cam.ac.uk; jordi.marti@upc.edu

\begin{abstract}
Metal cations are ubiquitous components in biological environments and play an important role in regulating cellular functioning and membrane properties. By applying metadynamics simulations, we have performed systematic free-energy calculations of $\mathrm{Na}^{+}, \mathrm{K}^{+}, \mathrm{Ca}^{2+}$, and $\mathrm{Mg}^{2+}$ bound to phospholipid membrane surfaces for the first time. The free-energy landscapes unveil specific binding behaviors of metal cations on phospholipid membranes. $\mathrm{Na}^{+}$and $\mathrm{K}^{+}$are more likely to stay in the aqueous solution, and can easily bind to a few lipid oxygens by overcoming low free-energy barriers. $\mathrm{Ca}^{2+}$ is most stable when bound to four lipid oxygens of the membranes, rather than being

${ }^{*}$ To whom correspondence should be addressed

${ }^{\dagger}$ Technical University of Catalonia-Barcelona Tech

${ }^{\ddagger}$ Boston University

ฯ University of Cambridge
\end{abstract}


hydrated in the aqueous solution. $\mathrm{Mg}^{2+}$ is tightly hydrated, and can hardly lose a hydration water and bind directly to the membranes. When bound to the membranes, the cations' most favorable total coordination numbers with water and lipid oxygens are the same as their corresponding hydration numbers in aqueous solution, indicating a competition between ion binding to water and lipids. The binding specificity of metal cations on membranes is then highly correlated with the hydration free-energy and the size of the hydration shell.

\section{INTRODUCTION}

Specific ion effects on biological systems have drawn great attention during last decades. ${ }^{1}$ In general, specific effects for cations are less pronounced than those for anions when ionwater interactions are dominant. ${ }^{2}$ However, for biological membranes in physiological environments, the interactions between metal cations and charged lipid headgroups are also essential and significant. Metal cations bound to membranes have been found to regulate membrane properties and membrane functioning, and such regulations deeply depend on the

ion specificity. ${ }^{3,4}$ Therefore, the understanding of specific interactions of metal cations with membranes is of great fundamental importance.

Several experiments suggest that metal cations are bound to the phosphate and carbonyl regions of phospholipid membranes. ${ }^{5-8}$ While bound to membranes in aqueous solution, a hydrated metal cation will lose one or more water molecules from its first hydration shell, resulting in several possible bound configurations. ${ }^{9,10}$ Although the binding constant and Gibbs free-energy of metal cation can be estimated from experiments, ${ }^{9}$ detailed studies of the relative stabilities of different bound states are difficult. Molecular dynamics (MD) simulations have been widely employed to investigate the role of metal cations in solvated phospholipid membranes from an atomic point of view. ${ }^{8,10-16}$ Nevertheless, the theoretical study of ionic binding to membranes is a computationally demanding task due to the long simulation times required to probe ion-membrane association and dissociation events. In 
addition, various bound configurations are separated by high free-energy barriers, making it difficult for MD simulations to sample them adequately. ${ }^{17}$ Free-energy calculations using enhanced sampling techniques provide a method to address the problem. However, despite the significant progress of free-energy calculations achieved in recent years, ${ }^{18}$ to the best of our knowledge, the binding free-energy landscape of metal cations on membrane surfaces is still missing. This is partially due to the difficulty of applying appropriate sampling techniques to address the problem, and also because of the complexity of the membrane environments, making the determination of the proper collective variables a difficult challenge. ${ }^{19}$

In this work, we have performed a systematic free-energy calculation of the binding states of metal cations at phospholipid membrane surfaces. The specific interactions of biologically relevant cations $\mathrm{Na}^{+}, \mathrm{K}^{+}, \mathrm{Ca}^{2+}$, and $\mathrm{Mg}^{2+}$ with membranes are revealed and interpreted from a free-energy perspective. The competition between ion-water and ion-membrane binding is also studied. Although such competition has been discussed before, ${ }^{12,14}$ here we provide for the first time a quantitative free energy characterization of the relevant ion binding states to water and lipids. Our work provides a general methodology to explore the free-energy landscapes for ions at complex biological interfaces which can be extended to study other interactions of interest between ions and charged headgroups in colloidal chemistry and biology. ${ }^{20}$

\section{METHODS}

Applying proper enhanced sampling methods and defining proper collective variables (CVs) are essential for the success of free-energy calculations. Recently, Jämbeck et al. showed that missing an essential CV in the construction of free energy profiles for membrane partitioning of solute can lead to divergences on the permeability of solute. ${ }^{21}$ While the normal direction to the plane of the membrane, $Z$, has been typically adopted as the reaction coordinate for the calculation of solute partitioning ${ }^{21,22}$ and ionic permeation, ${ }^{23,24}$ the problem of ion 
binding to membranes is more complex. Membranes are composed of a large number of lipid molecules with numerous conformations, and each lipid molecule has several binding sites at its headgroup, making it a challenge to define proper CVs to describe the interaction between ions and membranes. Moreover, ions are hydrated by water molecules, and the hydration level depends on the locations of ions at the membrane surfaces. Therefore, considering the interactions between ions and both lipid and water molecules could be a rational way for CV definition. A number of experiments ${ }^{5-8}$ and simulation works ${ }^{8,10-16}$ have shown that metal cations directly coordinate with the oxygen atoms of phosphate $\left(\mathrm{PO}_{4}^{-}\right)$and carbonyl $(\mathrm{C}=\mathrm{O})$ groups of lipid molecules, indicating that the oxygens in the lipid headgroup are the binding sites for metal cations. Accordingly, in this work we defined two CVs to describe the ionmembrane binding: the coordination number between a metal ion and lipid oxygens (CLP), and the coordination number between a metal ion and water oxygens (CWT). The details of the definition of the coordination numbers are given in the Supporting Information.

Well-tempered metadynamics ${ }^{25}$ a variant of metadynamics ${ }^{17,26}$ capable of enhancing the sampling of coordination numbers in multiple CV dimensions, was employed to calculate the free-energy landscape of ion binding to the membrane. Four sets of two-dimensional (2D) well-tempered metadynamics simulations based on the above CVs were performed to calculate the free-energy surfaces of $\mathrm{Na}^{+}, \mathrm{K}^{+}, \mathrm{Ca}^{2+}$, and $\mathrm{Mg}^{2+}$ ions at neutral zwitterionic phospholipid membranes. CHARMM-GUI ${ }^{27,28}$ was employed to generate four sets of lipid bilayer systems. Each system consisted of 50 dimyristoylphosphatidylcholine (DMPC) lipid molecules, ${ }^{29,30} 2500$ TIP3P water molecules, ${ }^{31}$ a metal cation, and one or two $\mathrm{Cl}^{-}$anions to neutralize the system. All the simulations were performed using NAMD $2.9^{32}$ together with PLUMED2 plugin ${ }^{33}$ and the CHARMM36 force field. ${ }^{34}$ The simulation parameters are described in the Supporting Information. 

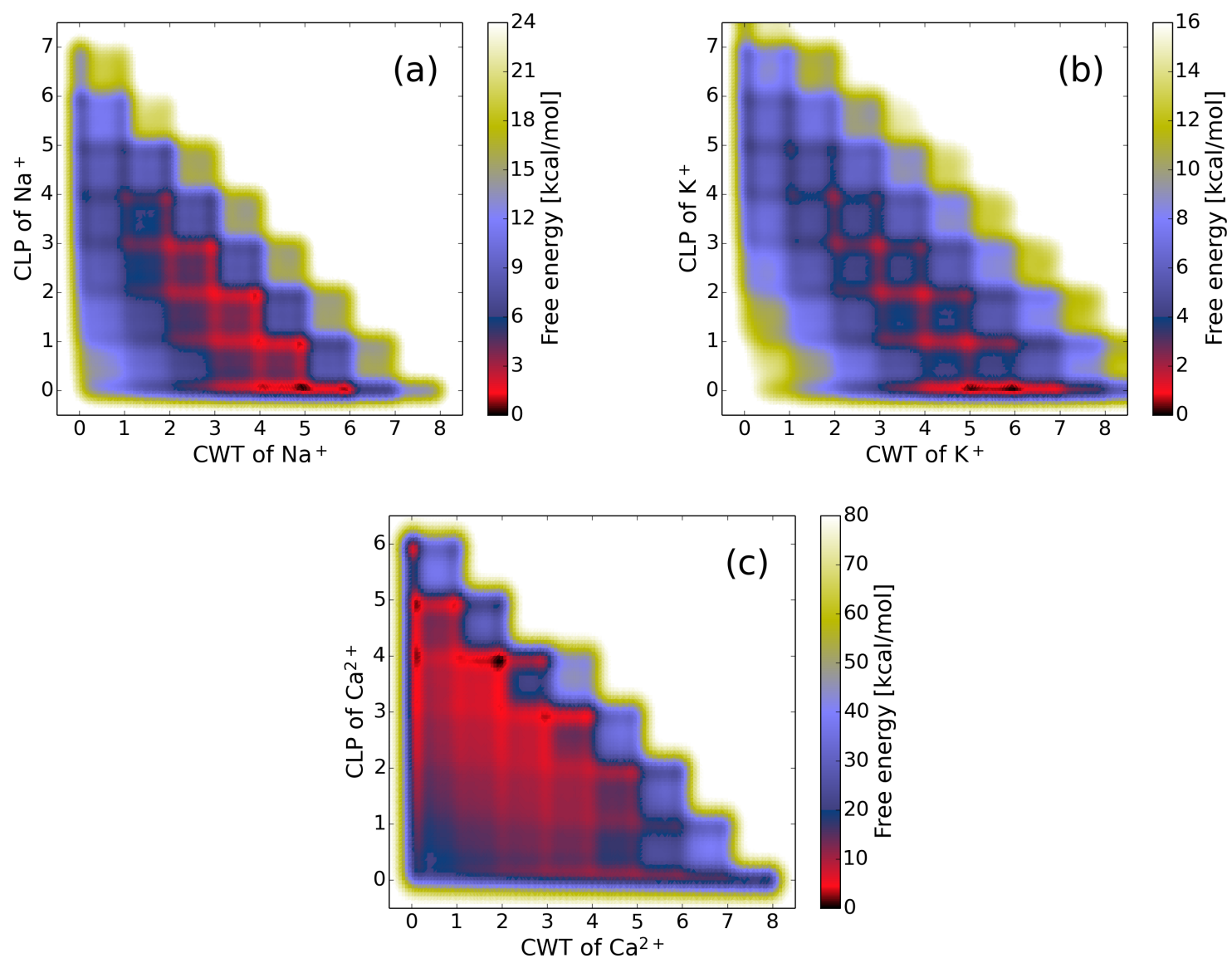

Figure 1: Free-energy surface as a function of the coordination number of water oxygens (CWT) and the coordination number of lipid oxygens (CLP) for $\mathrm{Na}^{+}(\mathrm{a}), \mathrm{K}^{+}(\mathrm{b})$, and $\mathrm{Ca}^{2+}$ (c) at DMPC membrane surfaces.

\section{RESULTS}

The resulting 2D free-energy surfaces (FES) of metal cations bound to DMPC membranes are shown in Figure 1. A staircase pattern is present in the FES of $\mathrm{Na}^{+}$in Figure 1a. The $\mathrm{Na}^{+}$ion is considered to be in the aqueous solution at CLP $=0$, where it can be hydrated by at most 8 water molecules. When it is fully dehydrated $(\mathrm{CWT}=0), \mathrm{Na}^{+}$can be bound to at most 7 lipid oxygens. Between these two extreme cases, $\mathrm{Na}^{+}$is bound to oxygens of both water and lipids. The fact that the states at local free energy minima are those with integer numbers of CLP and CWT validates our choice of CVs to explore the binding processes. The pattern shown in Figure 1a is a consequence of the competition between the 
binding of the ion to lipids and to water. Indeed, paths of approximately the same binding free energy are found for integer values of CLP and CWT with the same total coordination number $(\mathrm{CLP}+\mathrm{CWT})$. The global minimum of the FES is at the $(\mathrm{CWT}=5, \mathrm{CLP}=0)$ state, revealing that being hydrated by 5 water molecules in the aqueous solution is the most favorable state for $\mathrm{Na}^{+}$. When $\mathrm{Na}^{+}$is bound to the membranes, the stable bound states are located in the region with $\mathrm{CLP} \in[1,4]$ and $\mathrm{CWT} \in[1,5]$ with the total coordination number $(\mathrm{CLP}+\mathrm{CWT}) \in[4,6]$. A representative snapshot of a bound state of $\mathrm{Na}^{+}$coordinated with 6 oxygens is shown in Figure 2a.
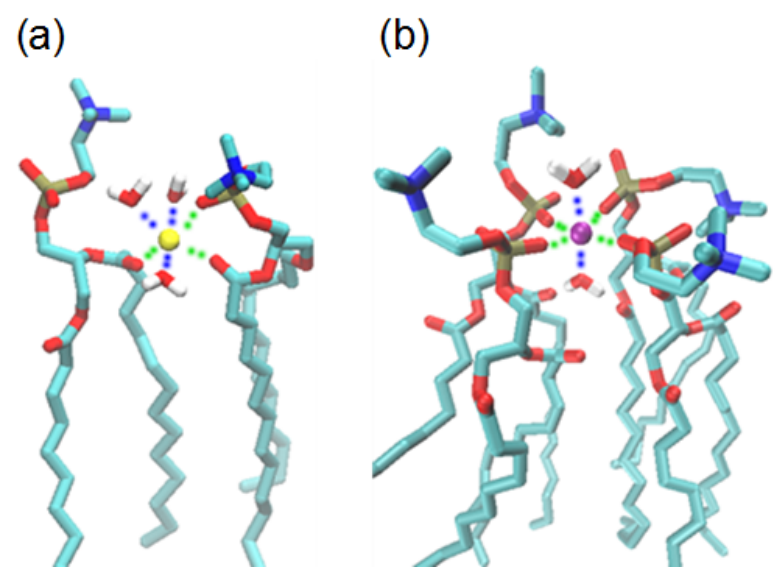

Figure 2: (a) A representative bound state for $\mathrm{Na}^{+}$coordinated to 3 lipid oxygens and 3 water molecules. (b) The most stable bound state for $\mathrm{Ca}^{2+}$ coordinated to 4 lipid oxygens and 2 water molecules. $\mathrm{Na}^{+}$is in yellow and $\mathrm{Ca}^{2+}$ is in purple. The binding to lipid oxygens and to water oxygens are shown in green and blue respectively.

Similar patterns are also present in the 2D FES of $\mathrm{K}^{+}$in Figure $1 \mathrm{~b}$, revealing similar binding behaviors of $\mathrm{K}^{+}$and $\mathrm{Na}^{+}$at aqueous membrane interfaces. Because of a larger atomic size, $\mathrm{K}^{+}$has a larger first coordination shell but a relatively lower surface charge density. The global minima states are at $(5,0)$ and $(6,0)$. Therefore, being hydrated by $5 \sim 6$ water molecules in the aqueous solution is most favorable for $\mathrm{K}^{+}$. When bound to membrane surfaces, $\mathrm{K}^{+}$can be coordinated to more water oxygens and lipid oxygens than $\mathrm{Na}^{+}$. The stable bound states for $\mathrm{K}^{+}$are in the region with $\mathrm{CLP} \in[1,4]$ and $\mathrm{CWT} \in[2,6]$ with the total coordination number $(\mathrm{CLP}+\mathrm{CWT}) \in[5,7]$.

While staying in the aqueous solution is most favorable for the monovalent metal cations, 
i.e. $\mathrm{Na}^{+}$and $\mathrm{K}^{+}$, being bound to the membrane surfaces is most stable for $\mathrm{Ca}^{2+}$. The global free-energy minimum at $(2,4)$ in Figure $1 \mathrm{c}$ indicates that $\mathrm{Ca}^{2+}$ is preferably bound to 4 lipid oxygens (see Figure 2b), rather than being hydrated in the aqueous solution. In contrast to the stable bound states of $\mathrm{Na}^{+}$and $\mathrm{K}^{+}$with $\mathrm{CLP} \in[1,4]$, those for $\mathrm{Ca}^{2+}$ are located in the region with $\mathrm{CLP} \in[2,6]$, which suggests a significantly greater affinity of $\mathrm{Ca}^{2+}$ to lipid oxygens than that of monovalent metal cations.

The case of $\mathrm{Mg}^{2+}$ is different from the other ions considered. The bound states of $\mathrm{Mg}^{2+}$ to lipid oxygens $(\mathrm{CLP} \geq 1)$ are separated from the unbound states in the aqueous solution $(\mathrm{CLP}=0)$ by high free-energy barriers, and could not be appropriately sampled by our calculations. This is attributed to the high energy required for partial dehydration of the first hydration shell of $\mathrm{Mg}^{2+}$, which is shown in Figure 3d, where only the case with CLP = 0 is considered. The strong binding of $\mathrm{Mg}^{2+}$ to its hydration layer has been also reported recently by Allnér et al. They calculated the potential of mean force between $\mathrm{Mg}^{2+}$ and water oxygens and that between $\mathrm{Mg}^{2+}$ and phosphate oxygens, and showed extremely high free-energy barriers for water dehydration and direct phosphate binding. ${ }^{35}$

Table 1: Free-energies for the lowest states of $\mathrm{Na}^{+}, \mathrm{K}^{+}$, and $\mathrm{Ca}^{2+}$ with respect to the global minimum.

\begin{tabular}{|c|c|c|c|c|}
\hline \multirow{2}{*}{ ion } & \multicolumn{3}{|c|}{ binding state } & \multirow{2}{*}{ F[kcal/mol $]$} \\
\cline { 2 - 5 } & CLP & CWT & CLP+CWT & \\
\hline $\mathrm{Na}^{+}$ & 0 & 5 & 5 & 0.0 \\
& 1 & 4 & 5 & 1.28 \\
& 2 & 3 & 5 & 1.69 \\
& 3 & 2 & 5 & 2.69 \\
\hline $\mathrm{K}^{+}$ & 0 & 6 & 6 & 0.0 \\
& 0 & 5 & 5 & 0.11 \\
& 1 & 5 & 6 & 1.23 \\
& 2 & 4 & 6 & 1.46 \\
& 3 & 3 & 6 & 1.78 \\
& 4 & 2 & 6 & 2.26 \\
\hline $\mathrm{Ca}^{2+}$ & 4 & 2 & 6 & 0.0 \\
& 4 & 0 & 4 & 2.07 \\
& 5 & 0 & 5 & 2.74 \\
& 3 & 3 & 6 & 3.13 \\
\hline
\end{tabular}



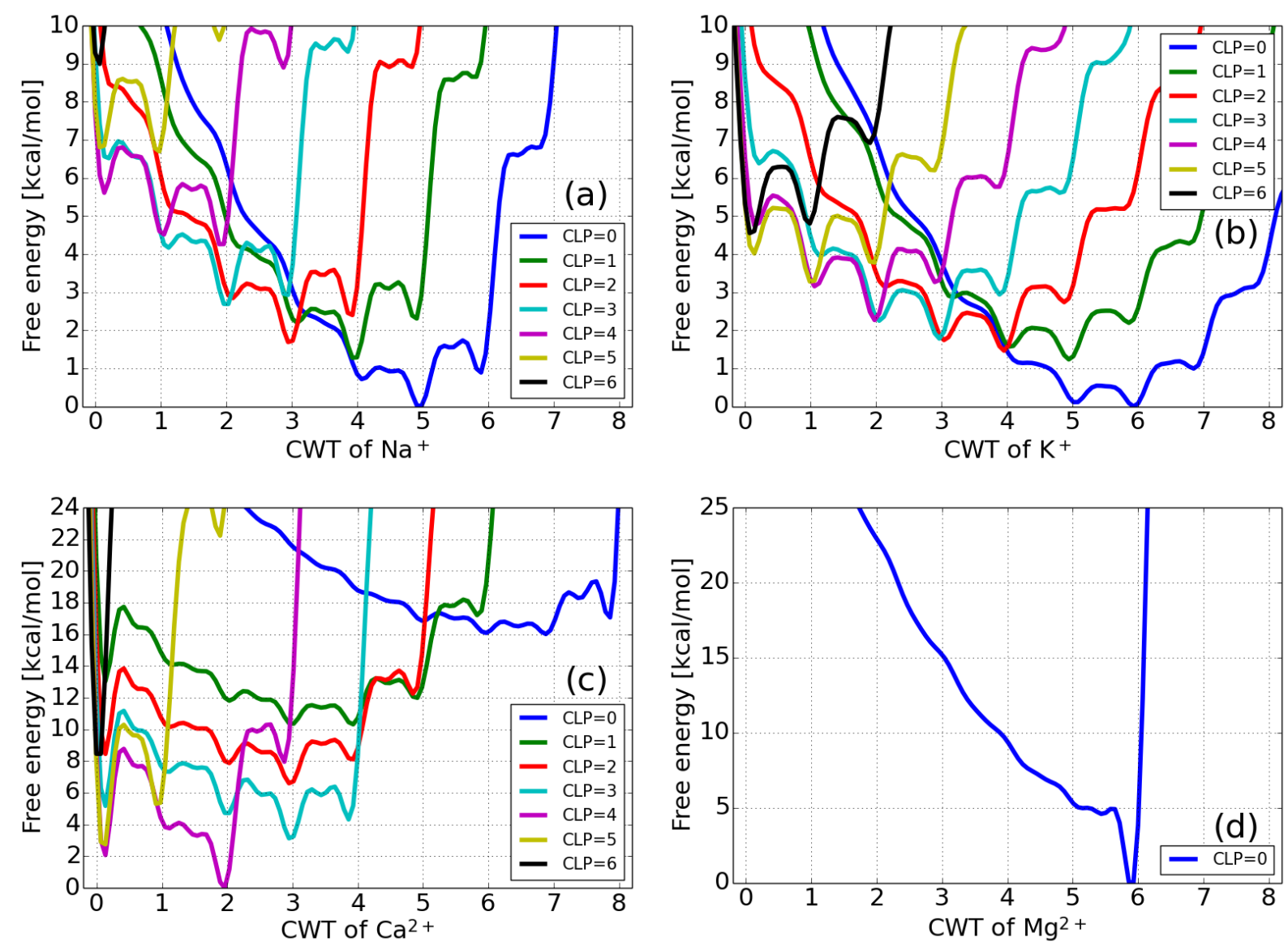

Figure 3: Free-energy as a function of CWT at various CLP for $\mathrm{Na}^{+}$(a), $\mathrm{K}^{+}$(b), $\mathrm{Ca}^{2+}$ (c) and $\mathrm{Mg}^{2+}(\mathrm{d})$. The data for $\mathrm{Na}^{+}, \mathrm{K}^{+}$, and $\mathrm{Ca}^{2+}$ are extracted from Figure 1. For $\mathrm{Mg}^{2+}$, only the case with CLP $=0$ is considered.

A quantitative representation of the above FES is given in Figure 3, where the freeenergies are plotted as a function of CWT at various CLP, and in Table 1, where the most relevant binding states are selected. As shown in Figure 3a, several hydration free-energy basins have been found for $\mathrm{CLP}=0$, where $\mathrm{Na}^{+}$is unbound to membranes. The most stable hydration state for $\mathrm{Na}^{+}$is the hydration with 5 water molecules, in agreement with the hydration number measured by experiments. ${ }^{36,37}$ The ion is considered bound to the membrane when CLP $>0$. For $\mathrm{Na}^{+}$at low binding, i.e. for CLP $=1,2,3$, the corresponding minimum free-energies are located at hydration levels $\mathrm{CWT}=4,3,2$, respectively, being 5 the total coordination number, which is the same as experimental hydration number. ${ }^{36,37}$ At high binding, i.e. for $\mathrm{CLP}=4,5,6$, the most stable hydration levels are CWT $=2,1$, 
0 , respectively, being 6 the total coordination number. The states with CLP $=1,2,3$ and total coordination number of $5 \sim 6$ are the lowest free-energy bound states, which are $1 \sim 2$ $\mathrm{kcal} / \mathrm{mol}$ higher than the global minimum at CLP $=0$. A quantitative characterization of the free-energies of those states is given in Table 1. Consequently, those are the most likely bound states of $\mathrm{Na}^{+}$that one is expected to find in a DMPC membrane immersed in a $\mathrm{NaCl}$ aqueous solution.

As shown in Figure 3b, the profiles for $\mathrm{K}^{+}$are similar to those for $\mathrm{Na}^{+}$. The hydration with 5 or 6 water molecules is most stable for $\mathrm{K}^{+}$at $\mathrm{CLP}=0$, in agreement with the experimental hydration number. ${ }^{36,37}$ Free-energy barriers constraining $\mathrm{K}^{+}$at the stable hydration states $(\mathrm{CWT}=5,6)$ are smaller than those for $\mathrm{Na}^{+}(\mathrm{CWT}=5)$, indicating a weaker affinity for water molecules of $\mathrm{K}^{+}$. At low binding, i.e. for CLP $=1,2,3$, the corresponding minimum free-energies are located at hydration levels $\mathrm{CWT}=5,4,3$, respectively, resulting in a total coordination number of 6 , which is the same as the experimental hydration number of $\mathrm{K}^{+} .36,37$ Note that the free-energy barriers for the lowest energy bound states of $\mathrm{K}^{+}$are also smaller than those for $\mathrm{Na}^{+}$, indicating an easier release from lipid binding sites.

While being hydrated in aqueous solution $(\mathrm{CLP}=0)$ is the most favorable state for $\mathrm{Na}^{+}$ and $\mathrm{K}^{+}$, for $\mathrm{Ca}^{2+}$ that state is $\sim 16 \mathrm{kcal} / \mathrm{mol}$ higher than the global minimum, as shown in Figure 3c. This shows that staying in the aqueous solution is greatly unfavorable for $\mathrm{Ca}^{2+}$ compared to being bound to the membranes. In aqueous solution, the most stable hydration states for $\mathrm{Ca}^{2+}$ are CWT $=6,7$, consistent with experimental hydration number. ${ }^{38,39}$ The most stable bound state for $\mathrm{Ca}^{2+}$ is at $(\mathrm{CWT}=2, \mathrm{CLP}=4)$, where the total coordination number is 6 . Other stable bound states are at CLP $=3,4,5$ with total coordination number of $4 \sim 6$, which are $2 \sim 4 \mathrm{kcal} / \mathrm{mol}$ higher than the global minimum (see Table 1 ). These bound states are also the global minima of the FES, which are $12 \sim 16 \mathrm{kcal} / \mathrm{mol}$ lower than the lowest energy unbound states at CLP $=0$. For $\mathrm{Ca}^{2+}$, we obtain a significantly stronger binding to phospholipid membranes over aqueous solution than for $\mathrm{Na}^{+}$and $\mathrm{K}^{+}$where the binding is weak, in agreement with experiment. ${ }^{7}$ For the ions considered, the ion binding free- 
energy has its minimum at the same total coordination number (CLP + CWT), irrespective of CLP or CWT, as shown in Table 1 and Figure S1 of the Supporting Information, and coincides with the hydration number in solution. This is a clear indication of the competition between ion binding to water and lipids, and is responsible for the correlation between ion hydration and ion binding specificity at the membranes.

Figure $3 \mathrm{~d}$ shows that in aqueous solution the hydration with 6 water molecules is the most stable state for $\mathrm{Mg}^{2+}$, in accordance with experiments. ${ }^{38,39}$ However, in contrast to the other cations which in solution have several hydration free-energy basins, $\mathrm{Mg}^{2+}$ has only one basin at $\mathrm{CWT}=6$, and the corresponding hydration free-energy barrier is very high $(\sim$ $5 \mathrm{kcal} / \mathrm{mol}$ ) compared with $\mathrm{Na}^{+}, \mathrm{K}^{+}$, and $\mathrm{Ca}^{2+}$. This is due to the strong binding of water molecules to $\mathrm{Mg}^{2+}$, which makes the change of its hydration state to other hydration states $(\mathrm{CWT}<6)$ and bound states $(\mathrm{CLP}>0)$ a rare event phenomenon.

\section{CONCLUSIONS}

In summary, we have provided a quantitative characterization of the binding states of biologically relevant metal cations $\left(\mathrm{Na}^{+}, \mathrm{K}^{+}, \mathrm{Ca}^{2+}\right.$, and $\left.\mathrm{Mg}^{2+}\right)$ at DMPC phospholipid membranes. With the help of well-tempered metadynamics simulations we have calculated the free-energies of the binding states, defined by the ion coordination to lipid (CLP) and water (CWT) oxygen atoms. Our results indicate that $\mathrm{Na}^{+}$and $\mathrm{K}^{+}$are more likely to stay in the aqueous solution, and can easily bind to $1 \sim 3$ lipid oxygens by overcoming free-energy barriers of $1 \sim 2 \mathrm{kcal} / \mathrm{mol}$. $\mathrm{Ca}^{2+}$ is most stable when bound to 4 lipid oxygens of the membranes, and the corresponding bound basin is $\sim 16 \mathrm{kcal} / \mathrm{mol}$ lower than the unbound states in the aqueous solution. $\mathrm{Mg}^{2+}$ has a strong affinity to hydration water, which makes the direct binding to the membranes difficult. When bound to the membranes, the cations' most favorable total coordination numbers with water and lipid oxygens are 5, 6, and 6 for $\mathrm{Na}^{+}$, $\mathrm{K}^{+}$, and $\mathrm{Ca}^{2+}$, respectively. Such coordination numbers coincide with their corresponding 
hydration numbers in bulk, suggesting a competition between ion binding to water and lipids.

\section{Acknowledgement}

The authors acknowledge computer resources and assistance provided by the Red Española de Supercomputación. J.Y. acknowledges support from a FI-AGAUR fellowship of the Generalitat de Catalunya. J.Y. and J.M. gratefully acknowledge financial support from the Direcció General de Recerca de la Generalitat de Catalunya (Grant 2009-SGR-1003) and the Spanish MINECO for grant FIS2012-39443-C02-01. C.C. acknowledges support from the Beatriu de Pinós program (BP-DGR 2011).

\section{Supporting Information Available}

Detailed simulation parameters, free-energy as a function of total coordination number $(\mathrm{CLP}+\mathrm{CWT})$, and free-energy convergence.

This material is available free of charge via the Internet at http://pubs.acs.org/.

\section{References}

(1) Lo Nostro, P.; Ninham, B. W. Chem. Rev. 2012, 112, 2286-2322.

(2) Kunz, W. Curr. Opin. Colloid Interface Sci. 2010, 15, 34-39.

(3) Berkowitz, M. L.; Bostick, D. L.; Pandit, S. Chem. Rev. 2006, 106, 1527-1539.

(4) Berkowitz, M. L.; Vácha, R. Acc. Chem. Res. 2012, 45, 74-82.

(5) Binder, H.; Zschörnig, O. Chem. Phys. Lipids 2002, 115, 39-61.

(6) Herbette, L.; Napolitano, C. A.; McDaniel, R. V. Biophys. J. 1984, 46, 677-685.

(7) Casillas-Ituarte, N. N.; Chen, X.; Castada, H.; Allen, H. C. J. Phys. Chem. B 2010, $114,9485-9495$. 
(8) Jurkiewicz, P.; Cwiklik, L.; Vojtíšková, A.; Jungwirth, P.; Hof, M. Biochim. Biophys. Acta - Biomembr. 2012, 1818, 609-616.

(9) Klasczyk, B.; Knecht, V.; Lipowsky, R.; Dimova, R. Langmuir 2010, 26, 18951-18958.

(10) Pandit, S. A.; Bostick, D.; Berkowitz, M. L. Biophys. J. 2003, 84, 3743-3750.

(11) Böckmann, R. A.; Hac, A.; Heimburg, T.; Grubmüller, H. Biophys. J. 2003, 85, 16471655.

(12) Böckmann, R. A.; Grubmüller, H. Angew. Chem., Int. Ed. 2004, 43, 1021-1024.

(13) Cordomí, A.; Edholm, O.; Perez, J. J. J. Phys. Chem. B 2008, 112, 1397-1408.

(14) Gurtovenko, A. A. J. Chem. Phys. 2005, 122, 244902.

(15) Gurtovenko, A. A.; Vattulainen, I. J. Phys. Chem. B 2008, 112, 1953-1962.

(16) Vácha, R.; Siu, S. W. I.; Petrov, M.; Böckmann, R. A.; Barucha-Kraszewska, J.; Jurkiewicz, P.; Hof, M.; Berkowitz, M. L.; Jungwirth, P. J. Phys. Chem. A 2009, 113, 7235-7243.

(17) Barducci, A.; Bonomi, M.; Parrinello, M. Wiley Interdiscip. Rev. Comput. Mol. Sci. 2011, 1, 826-843.

(18) Chipot, C. Wiley Interdiscip. Rev. Comput. Mol. Sci. 2014, 4, 71-89.

(19) Hansen, N.; Van Gunsteren, W. F. J. Chem. Theory Comput. 2014, 10, 2632-2647.

(20) Vlachy, N.; Jagoda-Cwiklik, B.; Vácha, R.; Touraud, D.; Jungwirth, P.; Kunz, W. Adv. Colloid Interface Sci. 2009, 146, 42-47.

(21) Jämbeck, J. P. M.; Lyubartsev, A. P. J. Phys. Chem. Lett. 2013, 4, 1781-1787.

(22) Wennberg, C. L.; Van Der Spoel, D.; Hub, J. S. J. Am. Chem. Soc. 2012, 134, 53515361. 
(23) Khavrutskii, I. V.; Gorfe, A. A.; Lu, B.; McCammon, J. A. J. Am. Chem. Soc. 2009, 131, 1706-1716.

(24) Vorobyov, I.; Olson, T. E.; Kim, J. H.; Koeppe, R. E.; Andersen, O. S.; Allen, T. W. Biophys. J. 2014, 106, 586-597.

(25) Barducci, A.; Bussi, G.; Parrinello, M. Phys. Rev. Lett. 2008, 100, 20603.

(26) Laio, A.; Parrinello, M. Proc. Natl. Acad. Sci. USA 2002, 99, 12562-12566.

(27) Jo, S.; Kim, T.; Iyer, V. G.; Im, W. J. Comput. Chem. 2008, 29, 1859-1865.

(28) Jo, S.; Lim, J. B.; Klauda, J. B.; Im, W. Biophys. J. 2009, 97, 50-58.

(29) Pasenkiewicz-Gierula, M.; Takaoka, Y.; Miyagawa, H.; Kitamura, K.; Kusumi, A. J. Phys. Chem. A 1997, 101, 3677-3691.

(30) Yang, J.; Calero, C.; Martí, J. J. Chem. Phys. 2014, 140, 104901.

(31) Jorgensen, W. L.; Chandrasekhar, J.; Madura, J. D.; Impey, R. W.; Klein, M. L. J. Chem. Phys. 1983, 79, 926-935.

(32) Phillips, J. C.; Braun, R.; Wang, W.; Gumbart, J.; Tajkhorshid, E.; Villa, E.; Chipot, C.; Skeel, R. D.; Kalé, L.; Schulten, K. J. Comput. Chem. 2005, 26, 1781-1802.

(33) Tribello, G. A.; Bonomi, M.; Branduardi, D.; Camilloni, C.; Bussi, G. Comput. Phys. Commun. 2014, 185, 604-613.

(34) Klauda, J. B.; Venable, R. M.; Freites, J. A.; O'Connor, J. W.; Tobias, D. J.; Mondragon-Ramirez, C.; Vorobyov, I.; MacKerell, A. D.; Pastor, R. W. J. Phys. Chem. $B$ 2010, 114, 7830-7843.

(35) Allnér, O.; Nilsson, L.; Villa, A. J. Chem. Theory Comput. 2012, 8, 1493-1502. 
(36) Mancinelli, R.; Botti, A.; Bruni, F.; Ricci, M. A.; Soper, A. K. J. Phys. Chem. B 2007, 111, 13570-13577.

(37) Varma, S.; Rempe, S. B. Biophys. Chem. 2006, 124, 192-199.

(38) Peschke, M.; Blades, A. T.; Kebarle, P. J. Phys. Chem. 1998, 102, 9978-9985.

(39) Bush, M. F.; O’Brien, J. T.; Prell, J. S.; Wu, C. C.; Saykally, R. J.; Williams, E. R. J. Am. Chem. Soc. 2009, 131, 13270-13277. 


\section{For Table of Contents use only.}

Title: Specific Ion Binding at Phospholipid Membrane Surfaces

Authors: Jing Yang, Carles Calero, Massimiliano Bonomi, Jordi Martí.

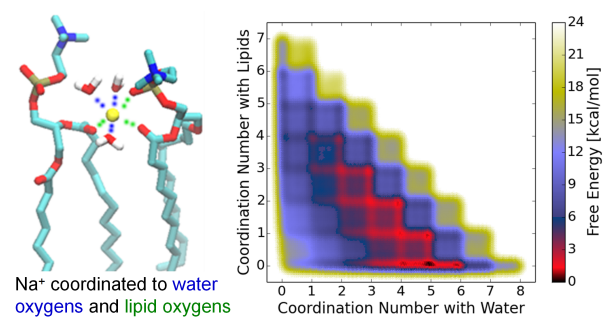

Figure 4: Table of Contents Graphic 